\title{
Neuromagnetic Activity During Recognition of Emotional Pictures
}

\author{
Johanna Kissler • Anne Hauswald
}

Accepted: 14 February 2008/Published online: 12 March 2008

(C) Springer Science+Business Media, LLC 2008

\begin{abstract}
Recently studied 'old' stimuli lead to larger frontal and parietal ERP responses than 'new' stimuli. The present experiment investigated the neuromagnetic correlates (MEG) of this 'old-new' effect and its modulation by emotional stimulus content. Highly arousing pleasant, highly arousing unpleasant and un-arousing neutral photographs were presented to the participants with the instruction to memorize them. They were later re-presented together with new photographs in an old-new decision task. In line with previous ERP studies, a long-lasting old-new effect (350-700 ms) was found. Independently, an emotion effect also occurred, as reflected in a, particularly left temporal, activity increase for emotional pictures between 450 and $580 \mathrm{~ms}$. Moreover, only for the pleasant pictures did the early part of the old-new effect, which is thought to reflect familiarity based recognition processes, interact with picture content: The old-new effect for pleasant pictures in frontal regions was larger than the one for neutral or unpleasant pictures between 350 and $450 \mathrm{~ms}$. In parallel, subjects' responses were accelerated towards and biased in favour of classifying pleasant pictures as old. However, when false alarm rate was taken into account, there was no significant effect of emotional content on recognition accuracy. In sum, this MEG study demonstrates an effect of particularly pleasant emotional content on recognition memory which may be mediated by a familiarity based process.
\end{abstract}

J. Kissler $(\bowtie) \cdot$ A. Hauswald

Department of Psychology, University of Konstanz, D25,

Konstanz 78457, Germany

e-mail: Johanna.Kissler@uni-konstanz.de
Keywords Emotion - Recognition memory · Old-new effect · Magnetoencephalography · International Affective Picture System

\section{Introduction}

From an evolutionary viewpoint, emotions serve adaptive functions, optimizing information processing and behaviour to increase survival chances. Therefore it is necessary that survival-relevant information be processed in a preferential way [10]. Ultimately, emotionally arousing stimuli that are associated with survival (threat, reproduction, food intake) serve to mobilize two motivational systems, an approach and an avoidance system [4, 28]. While behavioural output in response to appetitive and aversive stimuli is fundamentally different, humans preferentially attend to both pleasant and aversive stimuli alike. Electrophysiologically, this preferential attention to emotion is evident from earliest stages of stimulus registration [26], but extends into later time windows supposedly reflecting memory-associated processes [42]. Indeed, enhanced attention to a stimulus often results in better memory. Memory for emotional episodes and stimuli is a vital part of adaptive information processing as it allows for behavioural changes on the basis of experience with threatening or pleasurable objects or situations. Consequently, we remember emotionally arousing events such as a first kiss or the first funeral we attended more vividly than ordinary, emotionally neutral events.

In line with this every-day experience, memory research has shown that free recall of emotionally arousing experimental material (e.g. words, faces or pictures) is enhanced compared to neutral material. For instance, emotionally arousing pictures are recalled more often regardless of 
whether they were merely viewed [38] or also rated [2, 11, 12] during encoding. This effect is remarkably long lasting: Bradley and colleagues [2] showed that arousing pictures were recalled better than un-arousing ones not only shortly after encoding, but even at a delayed recall a year later. Memory enhancement for emotional material has also been found to be reflected in superior recognition memory for emotional stimuli [2] and long-term enhancement of recognition memory for pleasant and unpleasant pictures has been confirmed in a recent fMRI study [13]. However, particularly for recognition memory, several studies report no beneficial effect of emotion on either recognition hit rate $[18,32]$ or recognition accuracy (hits-false alarms, [33, $40,53])$. Thus, better recognition of emotional items may be due to a shift in response criterion, 'an emotion-induced recognition bias', rather than an actual superior discriminative recognition memory $[8,30,53]$. On the other hand, Ochsner [36] reported genuinely better discrimination particularly for unpleasant than for neutral pictures. Specifically, Ochsner's [36] results indicate that unpleasant and pleasant material selectively affects different aspects of recognition memory, pleasant material impacting the familiarity component and unpleasant material enhancing recollection.

A well-known ERP correlate of recognition memory is the old-new effect [e.g. 41]. It has already been investigated in several studies on emotional memory [23, 32, 45, 53]. The effect refers to the phenomenon of more positivegoing ERPs for correctly classified items that have been learned during a previous study phase compared to correctly 'rejected' (i.e. correctly identified as new) novel items during a recognition test. It emerges about $300 \mathrm{~ms}$ post stimulus and continues for several hundreds of milliseconds. In the ERP the old-new effect can be sub-divided into two spatially and functionally distinct stages: An early, frontally dominant phase, from about 300-500 ms after stimulus onset, which is thought to reflect familiarity-based processes and a later phase from about $500 \mathrm{~ms}$ onwards, associated with recollection [34].

Inaba and colleagues [23] investigated emotional modulation of the old-new effect with pleasant, unpleasant and neutral words and found that, from $400 \mathrm{~ms}$ onwards, overall the positivity was greatest for unpleasant hits. Pleasant hits were moderately more positive compared to neutral hits. Assessing recognition memory for emotional and neutral faces, Johansson and colleagues [24] found no overall difference in recognition performance for pleasant, unpleasant, and neutral faces. Yet, electrophysiological recordings revealed that the faces' emotional content affected brain responses both individually and in interaction with the old-new effect. Over frontal leads both pleasant and unpleasant faces were associated with more positive going waves, independently of the old-new effect.
However, an interaction of emotional content with the oldnew effect emerged in that unpleasant faces were associated with a larger parietal old-new effect, whereas pleasant faces were associated with a more pronounced frontal oldnew effect. In line with the interpretation of frontal oldnew effects as indicating familiarity based processes and of parietal old-new effects as indicating recollection based processes, a follow-up experiment confirmed that accurate recognition of pleasant and neutral items was predominantly based on a feeling of familiarity whereas performance for negative faces was based to a greater extent on recollection [24]. Behaviourally, these findings confirm Ochsner's results [36].

There are generally relatively few ERP studies that investigated the old-new effect using pictorial material. Those that did found the difference between old and new ERPs to be relatively more enhanced over frontal rather than posterior sites, both for pictures [17] and visual patterns [49].

The present study was designed to explore the impact of emotional stimulus content on the recognition memory for complex coloured photographs from the IAPS picture set [29]. The IAPS set has repeatedly been used to demonstrate preferential allocation of attention to emotionally arousing stimuli [26, 43], differential reflex modulation by emotional background stimuli [7] and behavioural indices of free-recall and recognition memory [2]. The predictiveness of a larger parietal positivity during encoding of emotional pictures for subsequent recall [11] as well as the impact of emotional material on working memory [39] have also been studied with IAPS pictures. Surprisingly however, so far no published study has investigated eletrophysiological (EEG) or neuromagnetic (MEG) mechanisms underlying the modulation of recognition memory as indexed by the old-new effect with stimuli from this otherwise well-used standardized picture set.

In the following, we will address the extent to which recognition memory for complex pictures as contained in the IAPS is modulated by emotional content using magnetoencephalography (MEG). So far, there are only very few studies using MEG to investigate the neural correlates of recognition memory. Tendolkar and colleagues [48] investigated MEG correlates of recognition memory for words. They found a neuromagnetic old-new effect between 400 and $1,000 \mathrm{~ms}$ post stimulus on-set which they accounted for with three individual dipoles, one in the left parietal lobe, one in the right parahippocampal gyrus and one in the right inferior frontal gyrus. All of these increased their activity in response to old versus new words. Walla et al. [50] used MEG to study level of processing (perceptual versus conceptual) effects in false recognition memory. In that study false alarms following deep versus shallow processing differed between 300 and $700 \mathrm{~ms}$ after word 
on-set. The difference was modelled as an activation increase in two bilateral posterior-temporal dipoles for false alarms following the deep encoding condition. Staresina et al. [47] investigated event-related magnetic fields related to recognition memory for words using the 'rememberknow' procedure. While a neuromagnetic correlate of the old-new effect is not reported, differences between neuromagnetic activity relating to recollection failure (misses), successful recollection (remember) and familiarity (know) based responses are shown: Both recollection- and familiarity-based as compared to missed responses were associated with enhanced activity at left frontal MEG sensors between 300 and $500 \mathrm{~ms}$ after word on-set. A further MEG study of recognition memory analyzed induced oscillations [15]. Düzel and colleagues [16] also investigated evoked magnetic responses reflecting novelty and familiarity in an associative recognition paradigm, where subjects had to learn and were tested on face-tool configurations. This latter study describes a fronto-parieto-temporal network with right frontal and parietal responses reflecting associative novelty and right superior temporal cortex exhibiting a familiarity driven response. The pattern is suggestive of a likely network subserving recognition memory for pictorial stimuli as revealed in the MEG, although differences between an associative and the presently used identity recognition task are to be expected. So far, results from MEG and EEG studies converge in that in recognition memory tasks memory-related brain activity is robustly seen from about $300 \mathrm{~ms}$ after stimulus on-set.

Like EEG, MEG has an excellent temporal resolution. Using a whole-head neuromagnetometer and inverse modelling as a complementary view of the data, improved spatial information can be provided. Because MEG is reference-free, much less sensitive to volume conduction effects than EEG and selectively picks up tangential sources from cortical sulci while omitting their radial components, particularly for cortical sources, MEG's spatial resolution is superior to the one of EEG. Also, potentials with predominantly radial components are often generated in deeper structures In the following we examine cortical activities during encoding and recognition as measured by MEG as well as recognition accuracy and reaction times for old and new highly arousing pleasant and unpleasant and un-arousing neutral pictures. We specifically assess to what extent picture valence or arousal affect early, familiarity-based, and late, recollection-based, parts of the neuromagnetic old-new effect. We use the L2Minimum-Norm Estimate (L2-MNE, e.g. [20]) to estimate the $2 \mathrm{D}$ spatial distribution of the underlying neuronal generators. Although the L2-MNE does not provide information in 3D space, it uses the full information contained in the data without necessitating additional anatomical or mathematical constraints [21, 22].

\section{Methods}

\section{Participants}

Fifteen student subjects (nine male, six female) with a mean age of 27.6 participated in this study. All participants were right-handed as determined by a German version of the Edinburgh Handedness Questionaire [37]. They received a payment of 7 Euros or course credit in return for participation. The data of two male and one female subject had to be excluded from analysis due to poor data quality, yielding twelve datasets for analysis. All subjects provided informed consent.

\section{Material}

The stimulus material comprised a total of 576 pictures, 192 per valence category (pleasant, unpleasant, and neutral). Most of the pictures were from the International Affective Picture System (IAPS, [29]), but some pictures were added to expand the contents of all categories and particularly to replace neutral single object pictures with more complex scenes and pictures containing more social contents. Ratings of valence and arousal based on the self-assessment manikin scale [2] were available for all pictures. Pleasant pictures (babies, puppies, sports, erotic scenes) had mean valence ratings of 6.60 (SD: 0.69) and mean arousal ratings of 5.10 (SD: 1.13), unpleasant pictures (mutilations, war scenes, disgusting scenes) were rated on average 2.55 (SD: 0.72) on valence and 5.93 (SD: 0.89) on arousal and neutral pictures (faces, bureau scenes, neutral social scenes) had mean valence ratings of 5.21 (SD: 0.57) and mean arousal ratings of 2.83 (SD: 0.87 ). The three emotion categories differed significantly in their valence and arousal values with the unpleasant pictures being the most arousing and least pleasant, the pleasant pictures being medium arousing and most pleasant, and the neutral pictures being least arousing and medium pleasant (all ps $<.05$ ). Pictures from the different valence categories (pleasant, neutral, unpleasant) were digitally matched for brightness and contrast. ${ }^{1}$ They all had a size of $640 \times 480$ pixels.

\footnotetext{
${ }^{1}$ Brightness was defined as the mean over all pixels of the blackwhite-brightness (mean of red, green, and blue intensities). Contrast was defined as the standard deviation of this brightness. Brightness and contrast were adjusted linearly, brightness of all pixels was in- or decreased by constant values (brightness) and brightness variance of the pixel from the mean increased by a factor (contrast). In case of ceiling or floor effects (brightness bigger than 255 or smaller than 0 ) adjustment was done by sinus scaled factors in dependence of the initial value. Thus, the more distant a value was from the boundary, the more it was adjusted. Therefore, the order of the brightness of the pixel remained while the values near the boundaries were compressed so that no ceiling or floor effects would occur (uniform black or white areas). This was done iteratively until the specified brightness and contrast values were reached.
} 
Setting

The experiment took place in a magnetically shielded chamber (Vacuumschmelze, Hanau, BRD) using a 148channel whole head magnetometer (Magnes $2500 \mathrm{WH}, 4 \mathrm{D}$ Neuroimaging, San Diego, USA). During measurements subjects lay comfortably inside the magnetically shielded room. Subjects lay supine and the visual stimuli were presented onto a white plastic screen attached to the ceiling of the room using a mirror projector system. Pictures subtended a visual angle of $10^{\circ}$ horizontally and $8^{\circ}$ vertically to either side from the centre of the screen.

\section{Procedure}

The experimental set-up is schematically depicted in Fig. 1. The experiment consisted of eight alternating encoding (1.26 $\mathrm{min})$ and test blocks $(2.16 \mathrm{~min})$ separated by a two minute break. In each encoding block thirty-six different critical pictures were presented for $900 \mathrm{~ms}$ with a $900 \mathrm{~ms}$ inter-stimulus interval. Additionally, three buffer pictures were added at the beginning and end of the block to counteract primacy and recency effects. Buffer pictures were not tested. Therefore the duration of the recognition block is less than twice the duration of the encoding block. Subjects were instructed to memorize the pictures while avoiding eye movements. After a 2-minute break the encoding phase was followed by a test block during which the 36 old pictures were presented in random sequence inter-mixed with 36 similar new pictures. As in the encoding phase, all pictures appeared for $900 \mathrm{~ms}$ with a $900 \mathrm{~ms}$ inter-stimulus interval during which a response was required. An old-new decision was made using the index and middle finger of the dominant hand. The finger response-category assignment was counterbalanced across subjects. Subjects were instructed to decide by button-press on a custom-made response pad as quickly and accurately as possible if a presented picture was old or new. In the inter-stimulus interval a fixation cross was presented in the encoding phase and a question mark in the test phase. The experiment was controlled using PRESENTATION software (Neurobehavioral Systems ${ }^{\circledR}$, Albany, NY, USA). Participants were asked to avoid eye movements and try to fixate the middle of the display during picture presentation.

\section{Data Recording and Analysis}

Neuromagnetic brain activity was recorded during both encoding and recognition with a sampling rate of $508.63 \mathrm{~Hz}$ and an online band-pass filter from $0.1 \mathrm{~Hz}$ to $100 \mathrm{~Hz}$. For artefact control, cardiac activity (electrocardiogram, ECG) as well as eye movements (electrooculogram, EOG) were recorded. ECG was recorded from two electrodes, one on each forearm, the EOG was measured from four electrodes attached above and below the right eye and to the left and right outer canthus of both eyes. For the EOG and ECG recordings a SynAmps amplifier (Neuroscan Laboratories, Sterling, VA, USA) was used.

Prior to the experiment, five index points on the subjects head (nasion, inion, left and right ear canal and CZ) and the subject's head shape were digitized with a Polhemus 3 Space $^{\circledR}$ Fasttrack. The participant's head position relative to the pick-up coils of the MEG was measured before and after each recording block.

The recorded MEG data was corrected for global noise by a correlative subtraction procedure as included in the 4D Neuroimaging ${ }^{\circledR}$ acquisition software using measurements from seven distant reference channels, three magnetometers and four gradiometers. Correction for eye movements and cardiac artefacts was run in Besa ${ }^{\circledR}$ (MEGIS Software $\mathrm{GmbH}$ ) using the in-built MSEC algorithm [1]. After artefact correction all epochs still containing signals $>3.5$
Fig. 1 The sequence of experimental events is depicted. Eight alternating encoding and test blocks were shown in immediate sequence. Each block contained different sets of pictures, 36 ( +6 buffer items) for learning and 72 (36 old and 36 new, randomly intermixed) for testing in the recognition phase

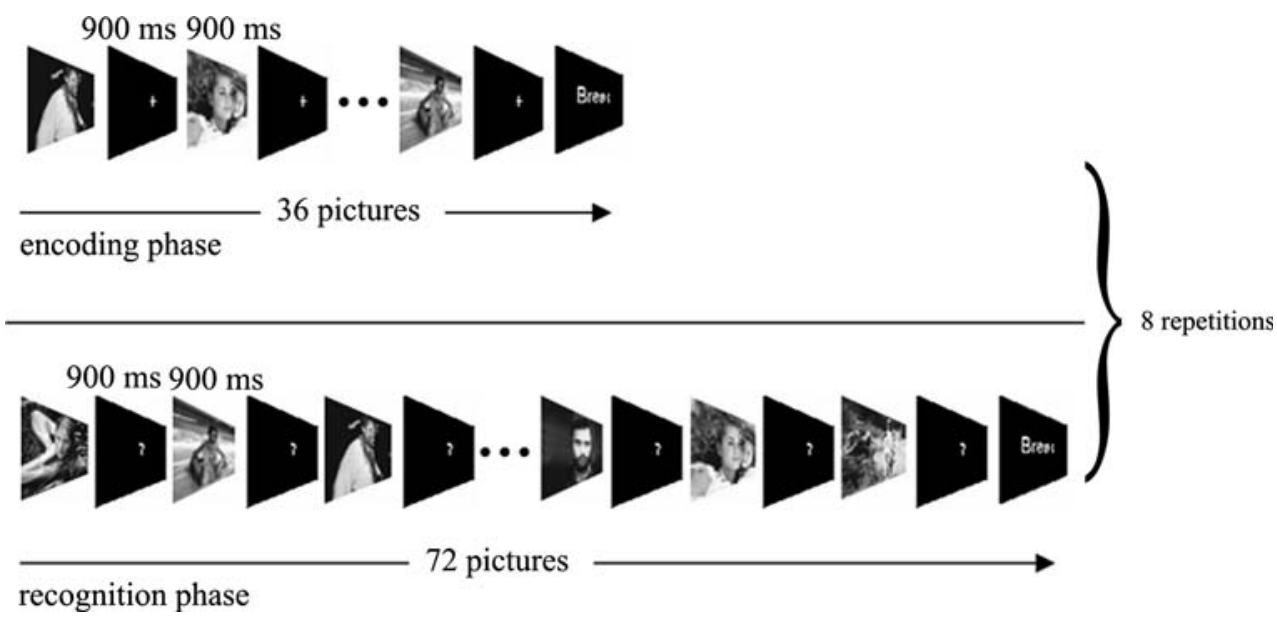


pT on one or more MEG channels were automatically rejected. The data were then divided into epochs from -100 to $900 \mathrm{~ms}$ and averaged. Using the Matlab-based software EMEGS $\subset$ ([25], http://www.emegs.org) the distribution of likely generators of the neuromagnetic activity was estimated using a L2 Minimum Norm Estimate (L2MNE; [20]). The L2-MNE served as an inverse distributed source modelling method to estimate the cortical generator structure of the magnetic field without a-priori assumptions. A four-shell isotropic spherical head model with 2 (azimuth and polar direction) $\times 197$ dipolar sources evenly distributed on a spherical shell was used. A radius of $6 \mathrm{~cm}$ was chosen as a best trade-off between depthsensitivity and spatial resolution [22]. The regularization parameter $\lambda$ was .02 and thus identical across all subjects and conditions. Topographies of dipole direction independent neural activities - the vector length of the generator activities at each position-were calculated for each individual subject, condition and time point based on the averaged magnetic field distributions and the individual sensor positions for each subject and run. For visualization purposes the estimated neural activities were projected onto the surface of a smoothened standard brain (see also $[27,44])$.

\section{Statistical Analysis}

For the behavioural reaction time and recognition rate data, two-way repeated measures ANOVAs with the factors Repetition (old-new) and Valence (pleasant, neutral, unpleasant) were calculated. Response biases (discrimination accuracy and recognition bias) were also assessed using a signal detection approach (see below). Post-hoc comparisons were calculated using Fisher's Least-Significant Difference tests.

The analyses of the neuromagnetic data focused on the old-new effect in the recognition blocks and only the correct response data due to few trials for meaningful averages of activities associated with incorrect responses. For the MEG data repeated-measures ANOVAs were calculated for the recognition phase with the factors Repetition (old, new) and Valence (pleasant, neutral, unpleasant). First, a step-wise ANOVA for each individual dipole location at each consecutive time-point $30 \mathrm{~ms}$ was run. The time course of the global field power of the F-values was used to determine the onset and duration of the old-new effect (see Fig. 5).

Within the thus determined time window of the neuromagnetic old-new effect, its spatial distribution, the impact of picture valence within this time window and the interaction of picture valence on the old-new effect were analysed. First F-maps depicting the time course and spatial distribution of significant main effects and interactions

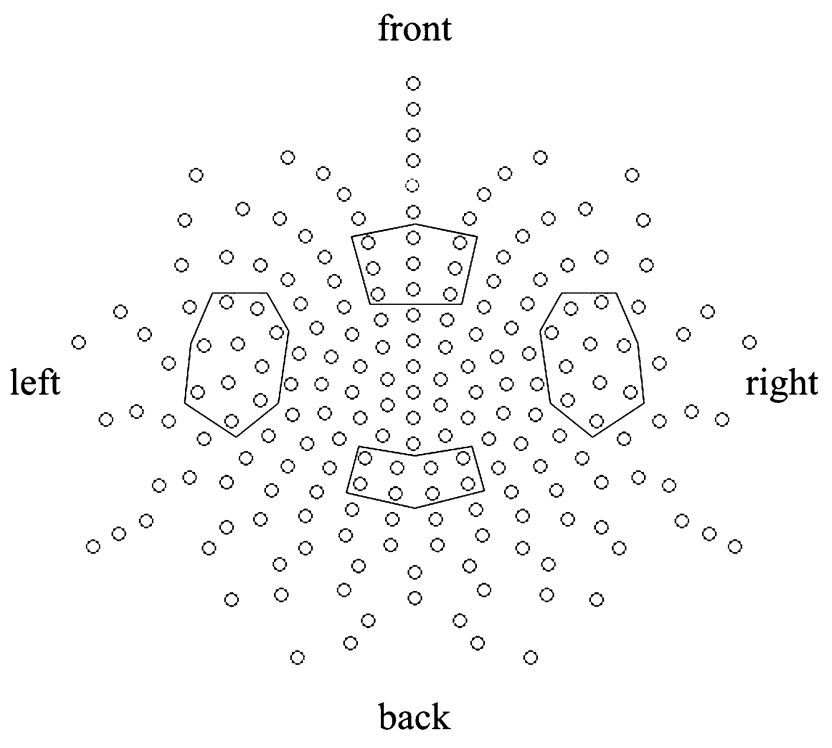

Fig. 2 Schematic layout of the dipole distribution used for the L2 Minimum-Norm Source Estimate. The dipole locations are arranged on a sphere and the figure shows a flattened rendering of the spherical configuration. The dipole groups (front, back, left, right) used for statistical analysis are marked

were generated in consecutive $30 \mathrm{~ms}$ windows (see Figs. 6-8). Second, more conservative confirmatory timewindow and regions of interest ANOVAs were conducted. Post-hoc comparisons were calculated using Fisher's Least-Significant Difference tests.

Four groups of dipoles were determined as regions of interest: a frontal, a posterior, a right, and a left group (see Fig. 2). Time windows of interest were determined on the basis of previous studies and by visual inspection of the morphology of the effects. Time windows chosen for the recognition phase were: $350-450 \mathrm{~ms}, 450-580 \mathrm{~ms}, 580$ $670 \mathrm{~ms}$.

\section{Results}

Behavioural Data

\section{Reaction Times}

Means and standard deviations (in brackets) for correct responses in the individual conditions were as follows: Pleasant Old: $712 \mathrm{~ms}$ (58.72). Neutral Old: $719 \mathrm{~ms}$ (62.18). Unpleasant Old: $725 \mathrm{~ms}$ (52.41). Pleasant New: 744 (57.68). Neutral New: 716 ms (46.62). Unpleasant New: 745 ms (61.80).

A two-way repeated measures ANOVA for the correct responses yielded a significant Valence main effect $[\mathrm{F}(2$, $22)=9.20, P<.01]$ indicating that overall both unpleasant $(P<.01)$ and pleasant $(P<.05)$ pictures were 


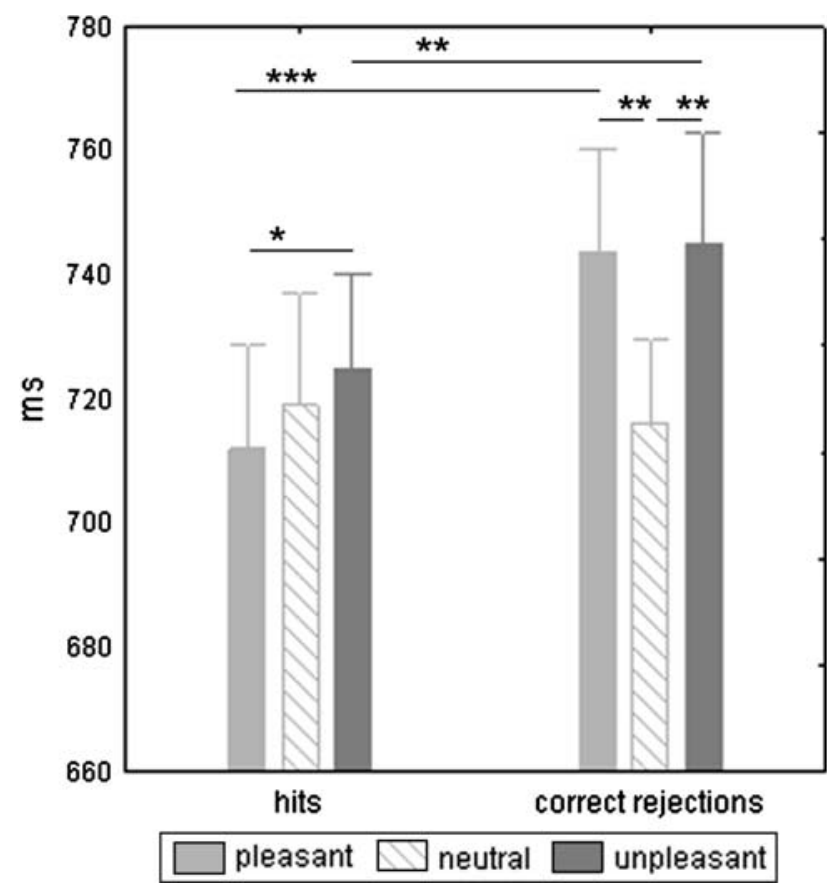

Fig. 3 Reaction times for correctly classified old pictures (hits) and new pictures correctly classified new pictures (correct rejections). Significant differences are indicated as follows: $* P<.05$; ** $P<.01 ; * * * P<.001$

responded to more slowly than neutral pictures. There was a trend-level effect of Repetition on the reaction times $[\mathrm{F}(2$, $22)=3.45, P<.1]$ and an interaction of both main effects [Repetition $\times$ Valence, $\mathrm{F}(2,22)=8.87, P<.01$, see Fig. 3]. While for neutral pictures correct rejections of new pictures were just as fast as correct classifications of old pictures, correct rejections of both pleasant and unpleasant pictures were slowed in comparison to the respective hits to

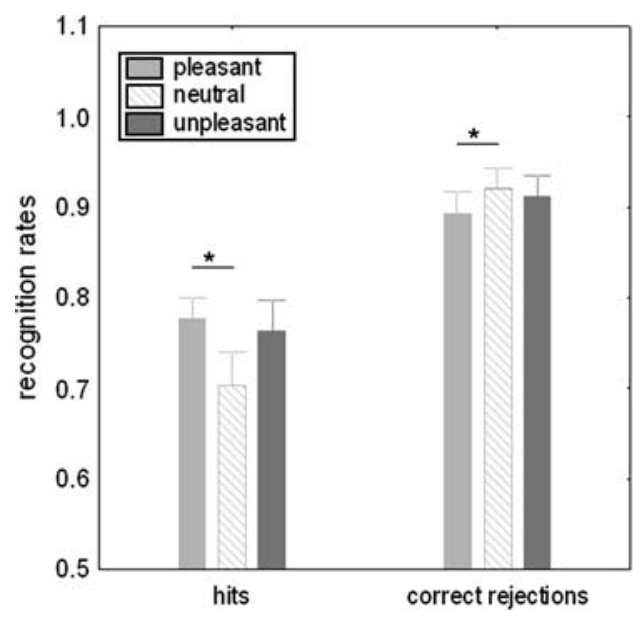

Fig. 4 Left: Probability for correct responses to old (hits) and new pictures (correct rejections). Right: accuracy for the old-new discrimination $(\mathrm{Pr})$ and response bias $(\mathrm{Br})$ for pleasant, neutral, and old pictures. Moreover, hits to pleasant pictures were fastest and particularly faster than hits to unpleasant pictures $(P<.05)$, whereas correct rejections were modulated by arousal and were slowed in comparison to neutral pictures for both pleasant and unpleasant (both $P<.01$ ).

\section{Recognition Rates}

The following recognition rates were obtained: Pleasant Old: .78 (.08). Neutral Old: .70 (.13). Unpleasant Old: .76 (.12). Pleasant New: .89 (.09). Neutral New: .92 (.09). Unpleasant New: .91 (.08).

A two-way (Repetition: old, new; Valence: pleasant, neutral, unpleasant) ANOVA of the correct recognition rates yielded a significant main effect of Repetition $[\mathrm{F}(1$, $11)=34.65, P<.001]$ with new pictures being more often correctly recognized as new than old pictures as old. The ANOVA also yielded a significant interaction Repetition $\times$ Valence $[\mathrm{F}(2,22)=5.28, P<.05$, Fig. 4]. Within the new pictures, neutral pictures were correctly rejected most often, the difference between neutral and pleasant pictures being significant $(P<.05)$. Within the old pictures particularly pleasant $(P<.05)$ and in tendency also unpleasant ones $(P<.1)$ were correctly recognized more often than neutral ones (see Fig. 4).

\section{Discrimination Accuracy and Recognition Bias}

The discrimination accuracy and recognition bias were analyzed to account for potential material-specific response tendencies. Discrimination accuracy of old and new pictures $\mathrm{Pr}$ (hits-false alarms) and the recognition bias $\mathrm{Br}$ (false alarms/(1-Pr)) were calculated according to the

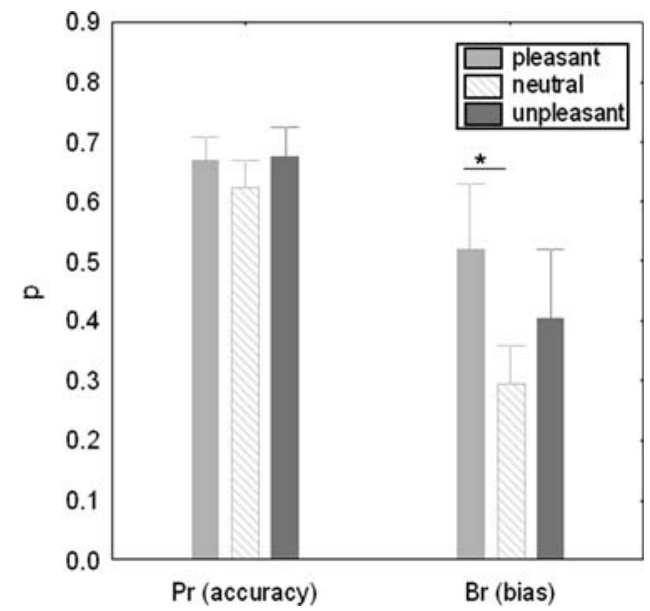

unpleasant pictures. Significant differences are indicated as follows: $* P<.05$ 
two-high-threshold model of Snodgrass and Corwin [46]. For hits and false alarms probability measures were used (see Fig. 4). The accuracy measure was somewhat, but not significantly higher for emotional pictures $[\mathrm{F}(2$, $22)=1.78, P=.19]$. However, overall recognition tended to be biased in favor of pleasant pictures $[\mathrm{F}(2,22)=3.24$,

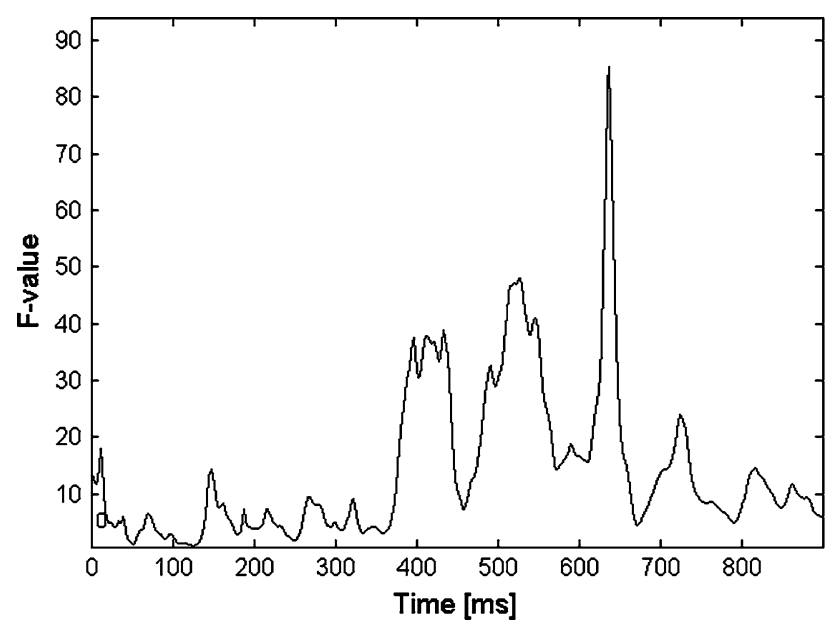

Fig. 5 Time course of the global field power of F-values for the repetition (old-new) main effect across all dipole locations at each time point. From this, the timing of the most significant effects can be gauged. Most significant old-new differences occur between about 350 and $650 \mathrm{~ms}$ after picture on-set
$P=.058]$ which were particularly more likely to be classified as old than neutral ones $(P<.05)$.

\section{MEG Data}

Figures 5 and 6 depict the temporal and spatial evolution of the old-new effect using F-maps from a point-wise ANOVA. Within the old-new effect, an independent significant impact of valence on cortical activity could be determined at frontal and predominantly left temporal sites as shown in the F-maps in Fig. 7. Moreover, there was also a period where emotional picture content and the old-new effect interacted. The time course and spatial extent of this interaction effect is shown in Fig. 8.

In line with the literature visual inspection of the F-maps (see Figs. 5 and 6) suggests that the old-new effect can be divided into several distinct stages. We identified three such stages, namely $350-450 \mathrm{~ms}$ were activity is predominantly frontal, $450-580 \mathrm{~ms}$, where activity is frontotemporal and 580-670 ms with predominantly temporoparietal activity. Across activation means in these selected time windows and regions confirmatory ANOVAs were calculated with the factors Repetition (old, new), Valence (pleasant, neutral, unpleasant), and Region (front, back, left, right). The results of this regions of interest ANOVA are detailed below.
Fig. 6 F-maps of the spatial and temporal distribution of the repetition (old-new) main effects in $30 \mathrm{~ms}$ steps between 330 and $700 \mathrm{~ms}$ after picture onset. The maps show an interpolated rendering of the statistical F-values at each source dipole projected onto a smoothed standard brain. Areas of significant differences are in red

\section{Old-new Effect (F-maps)}

$330.1 \mathrm{~ms}-359.5 \mathrm{~ms} 361.5 \mathrm{~ms}-391 \mathrm{~ms} 392.9 \mathrm{~ms}-422.4 \mathrm{~ms} 424.4 \mathrm{~ms}-453.8 \mathrm{~ms} 455.8 \mathrm{~ms}-485.3 \mathrm{~ms} 487.2 \mathrm{~ms}-516.7 \mathrm{~ms}$
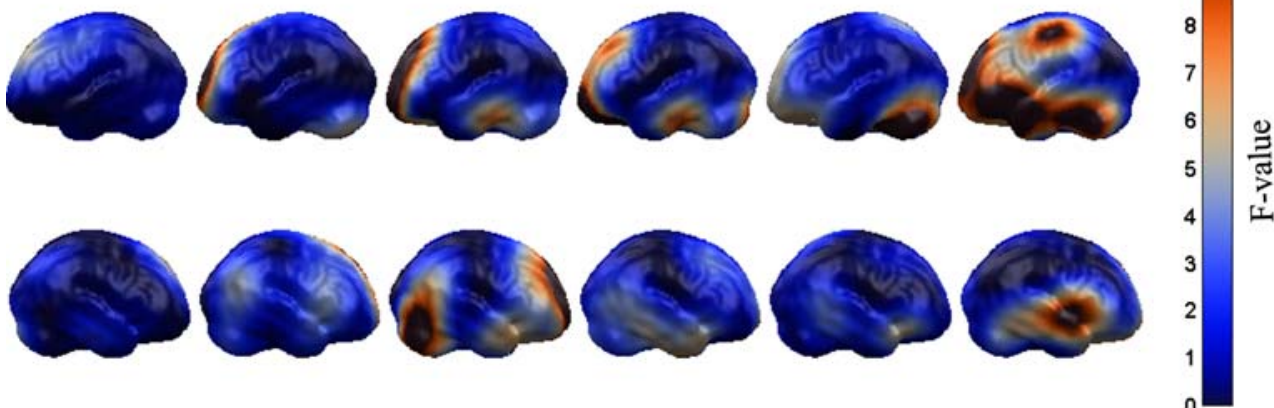

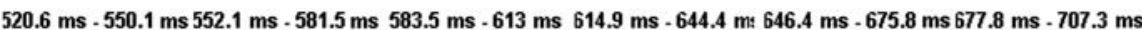
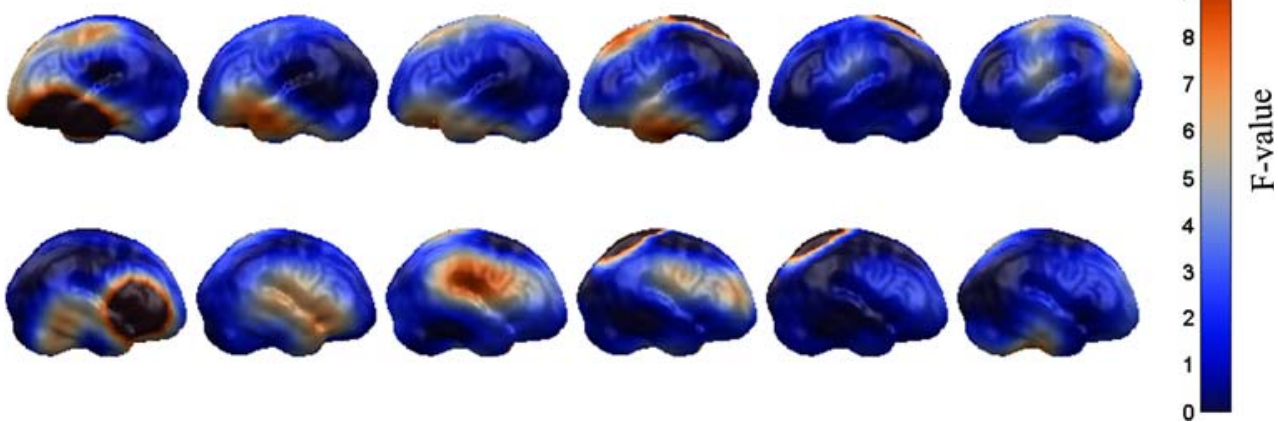
Fig. 7 F-maps of the spatial and temporal distribution of the valence main effects in $30 \mathrm{~ms}$ steps between 330 and $700 \mathrm{~ms}$ after picture onset. The maps show an interpolated rendering of the statistical F-values at each source dipole projected onto a smoothed standard brain. Areas of significant differences are in red

\section{Valence Effect (F-maps)}

$330.1 \mathrm{~ms}-359.5 \mathrm{~ms} 361.5 \mathrm{~ms}-391 \mathrm{~ms} 392.9 \mathrm{~ms}-422.4 \mathrm{~ms} 424.4 \mathrm{~ms}-453.8 \mathrm{~ms} 455.8 \mathrm{~ms}-485.3 \mathrm{~ms} 487.2 \mathrm{~ms}-516.7 \mathrm{~ms}$
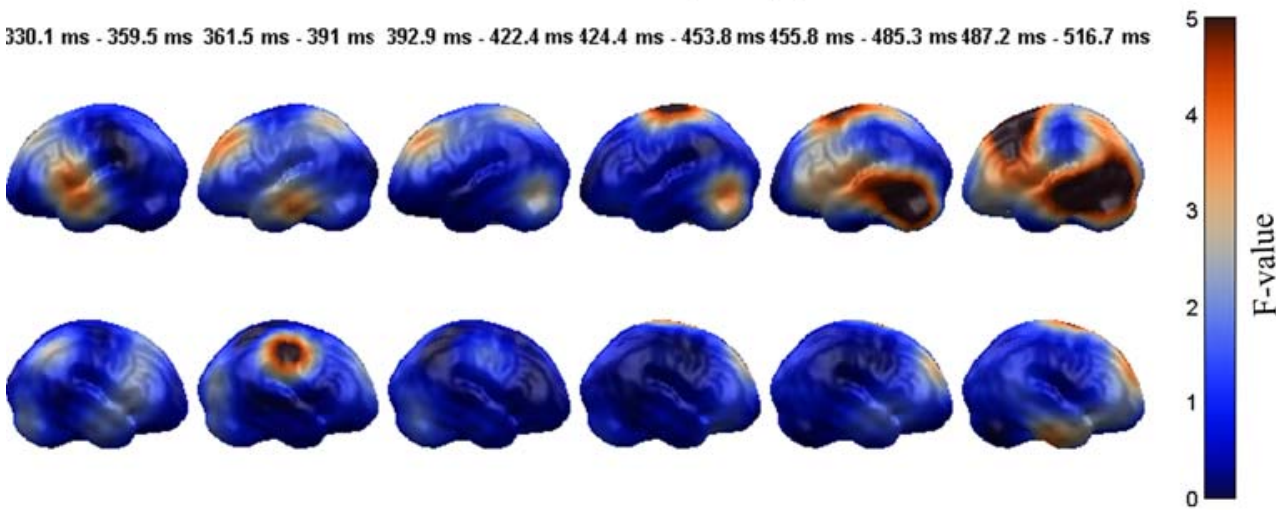

$520.6 \mathrm{~ms}-550.1 \mathrm{~ms} 552.1 \mathrm{~ms}-581.5 \mathrm{~ms} 583.5 \mathrm{~ms}-613 \mathrm{~ms} 614.9 \mathrm{~ms}-644.4 \mathrm{~ms} 646.4 \mathrm{~ms}-675.8 \mathrm{~ms} 677.8 \mathrm{~ms}-707.3 \mathrm{~ms}$
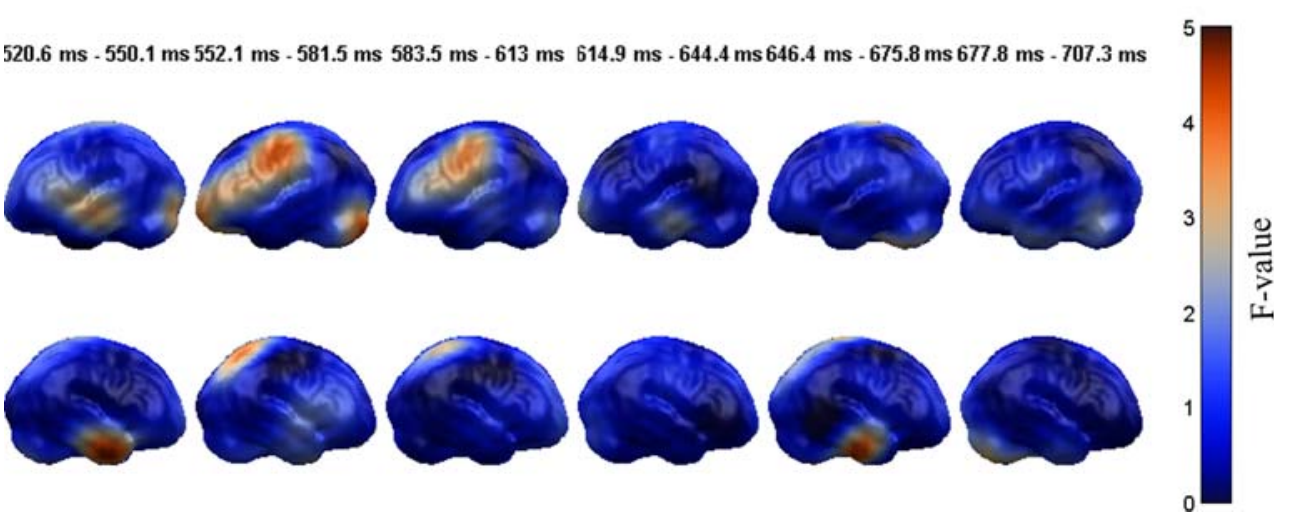

Interaction of repetition and valence (F-maps)

$330.1 \mathrm{~ms}-359.5 \mathrm{~ms} 361.5 \mathrm{~ms}-391 \mathrm{~ms} 392.9 \mathrm{~ms}-422.4 \mathrm{~ms} 424.4 \mathrm{~ms}-453.8 \mathrm{~ms} 455.8 \mathrm{~ms}-485.3 \mathrm{~ms} 487.2 \mathrm{~ms}-516.7 \mathrm{~ms}$
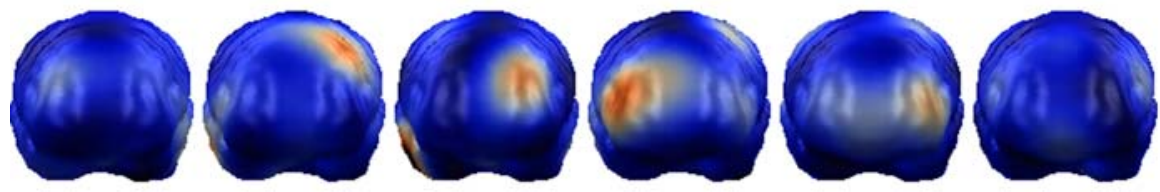

$520.6 \mathrm{~ms}-550.1 \mathrm{~ms} 552.1 \mathrm{~ms}-581.5 \mathrm{~ms} 583.5 \mathrm{~ms}-613 \mathrm{~ms} 614.9 \mathrm{~ms}-644.4 \mathrm{~ms} 646.4 \mathrm{~ms}-675.8 \mathrm{~ms} 677.8 \mathrm{~ms}-707.3 \mathrm{~ms}$
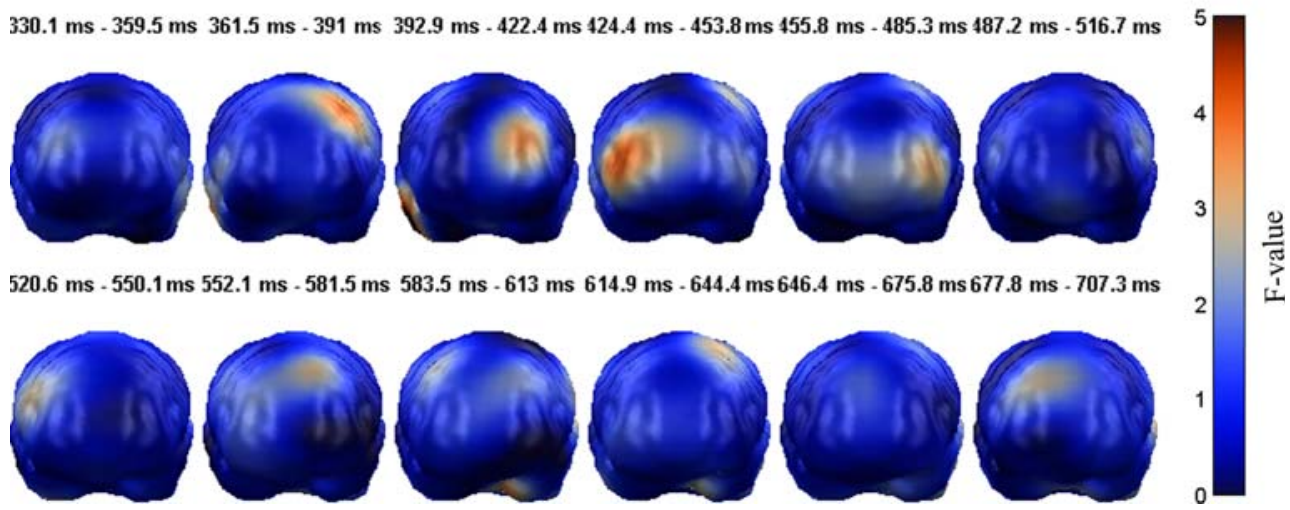

Fig. 8 F-maps of the spatial and temporal distribution of significant interactions between the 'old-new' and valence effects in $30 \mathrm{~ms}$ steps between 330 and $700 \mathrm{~ms}$ after picture onset. The maps show an

\section{$350-450 \mathrm{~ms}$}

A Region main effect $[\mathrm{F}(3,33)=8.26, \quad P<.001]$ occurred with less activity in the temporal regions compared to the anterior $(P<.05)$ and posterior ones $(P<.01)$. Also, a Repetition main effect $[\mathrm{F}(1$, $11)=11.185, P<.01$; see Figs. 5, 6, 9] and an interaction of Repetition and Region $[\mathrm{F}(3,33)=5.110, P<.01]$ interpolated rendering of the statistical F-values at each source dipole projected onto a smoothed standard brain. Areas of significant differences are marked in red

was found. The former reflected the MEG correlate of the well-known old-new effect with enhanced activity for old pictures and the latter showed that this old-new effect was greatest in frontal regions $(P<.001)$, medium in right lateral areas $(P<.05)$ and absent in posterior and left lateral sites. Figure 9 shows the difference between cortical activities evoked by old and new pictures for the three time-windows of interest. 
Fig. 9 Maps of differences of estimated cortical activation between old and new pictures in three different time windows. Shown is the interpolated difference of grand means of estimated cortical activity $\left(\mathrm{nAm} / \mathrm{cm}^{2}\right)$ evoked by old minus new pictures (modulus of cortical activity at each source dipole) projected onto a smoothed standard brain

\begin{tabular}{|c|c|c|c|c|c|}
\hline \multicolumn{6}{|c|}{ Old-new difference maps } \\
\hline & frontal & back & left & right & \\
\hline $\begin{array}{c}350-450 \\
\mathrm{~ms}\end{array}$ & & & & & हू \\
\hline $\begin{array}{c}450-580 \\
\mathrm{~ms}\end{array}$ & & & & & 䲻 \\
\hline $\begin{array}{c}580-670 \\
\mathrm{~ms}\end{array}$ & & & & & \\
\hline & & & & & \\
\hline
\end{tabular}

Additionally, a Repetition $\times$ Valence interaction $[\mathrm{F}(2$, $22)=5.29, P<.05]$ arose with the smallest old-new effect for neutral pictures $(P<.05)$, a somewhat stronger effect for unpleasant pictures $(P<.01)$, and the greatest effect for pleasant pictures $(P<.001)$ due to substantially increased activity for old pleasant pictures (see Figs. 8 and 10). Neither the Valence effect $[\mathrm{F}(2,22)=1.48$, $P=.25)]$, nor the Valence $\times$ Region $[\mathrm{F}(6,66)=1.42$, $P=.22]$ or Repetition $\times$ Valence $\times$ Region effect $[\mathrm{F}(6$, $66)=.34, \quad P=.51]$ reached significance. Figure 10 depicts the old-new effect in the different valence categories as the difference of cortical activation for old minus new pictures for each valence category. As can be seen from this difference plot, the impact of emotion on the old-new effect is primarily driven by a greater old-new effect for pleasant pictures.

\section{$450-580 \mathrm{~ms}$}

Again, reflecting the old-new effect, a significant impact of Repetition was found $[\mathrm{F}(1,11)=14.79, P<.01$; see Figs. 5, 6 and 9]. Emotional content tended to affect neural
Fig. 10 Maps of estimated cortical activation differences between old and new pictures separately for the three different emotion categories in the time window from $350-450 \mathrm{~ms}$ after picture onset. Shown is the interpolated difference of grand means of estimated cortical activity $\left(\mathrm{nAm} / \mathrm{cm}^{2}\right)$ evoked by old minus new pictures (modulus of cortical activity at each source dipole) projected onto a smoothed standard brain

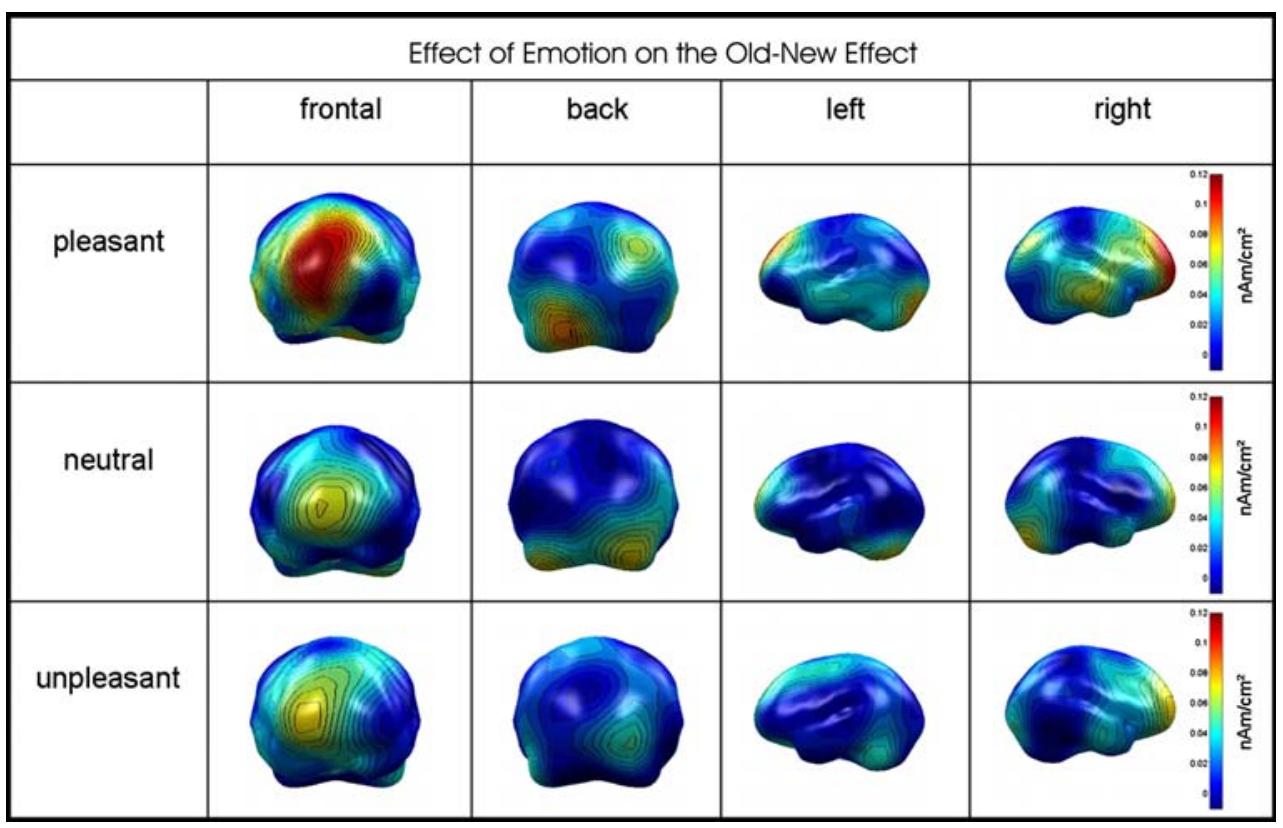


activity [Valence, $\mathrm{F}(2,22)=2.98, P<.07$, Fig. 7] and this effect differed between the regions of interest [Valence $\times$ Region, $\mathrm{F}(3,33)=2.44, P<.05$ ]. Post-hoc comparisons showed that in the left temporal region both pleasant and unpleasant pictures led to enhanced activity compared to neutral ones (both $P<.01$ ), while in the right temporal region only unpleasant pictures were associated with significantly enhanced activity compared to neutral ones $(P<.01)$. The pleasant-neutral comparison yielded only a marginal effect $(P=.07)$. In the frontal and parietal regions of interest the valence effect was not significant across the entire analysis window. However, the old-new effect had spread across all regions of interest [Region, $\mathrm{F}(3$, $33)=1.05, \quad P=.38$ and Repetition $\times$ Region, $F(3$, $33)=1.49, P=.23$ ] and did not interact with Valence [Repetition $\times$ Valence, $\mathrm{F}(2,22)=.37, P=.69$ and Repetition $\times$ Valence $\times$ Region, $\mathrm{F}(6,66)=.45, \quad P=.84]$. Figure 11 shows the difference in neural activity induced by emotional and neutral pictures between 450 and $580 \mathrm{~ms}$ after picture onset, irrespective of the repetition effect.

\section{$580-670 \mathrm{~ms}$}

A Repetition effect $[\mathrm{F}(1,11)=5.82, P<.05$, see Figs. 5 , 6 , 9] was found again. However neither the effect of Valence $[\mathrm{F}(2,22)=2.51, P=.10]$, nor the effect of Region $[\mathrm{F}(3,33)=.10, P=.96]$ was significant in this time window. Likewise, none of the interaction effects approached significance [Repetition $\times$ Valence, $F(2$, $22)=.01, P=.99$; Repetition $\times$ Region, $\mathrm{F}(3,33)=.78$, $P=.51 ;$ Valence $\times$ Region, $\mathrm{F}(6,66)=.53, P=.79$; Repetition $\times$ Valence $\times$ Region, $\mathrm{F}(6,66)=.78, P=.59]$.

\section{Discussion}

This study investigated emotional modulation of recognition memory for complex pictures and its underlying neuromagnetic correlates. In line with previous ERP studies, we found recognition memory related neuromagnetic activity to be enhanced between about 300 and $700 \mathrm{~ms}$ after stimulus on-set [19, 24, 34]. Our results are consistent with previous studies suggesting that a network of frontal, temporal and parietal regions exhibits enhanced activity during recognition of old versus new stimuli $[16,48]$. The present analysis suggests a temporal sequence of events with an early frontal effect, an intermediate fronto-temporal and a late parietal effect.

Going beyond previous studies, we analysed the modulation of the old-new effect by emotional picture content. Behaviourally, response speed towards and recognition of the presented pictures was influenced by emotional content: Responses to previously seen 'old' pleasant pictures were accelerated, particularly compared to unpleasant ones. Rejection of emotionally arousing pleasant and unpleasant 'new' pictures, by contrast, was generally slowed. While recognition performance initially also appeared to be modulated by emotional arousal, with seemingly more correct 'hits' being made to emotionally arousing old pictures, taking into account false alarms changed the picture: Discrimination accuracy for emotional pictures was no longer significantly improved, but a response bias in favour of pleasant pictures emerged.

Neuromagnetic data parallel and extend this pattern. They also deserve attention independent of their emotional modulation, this being one of the first studies to investigate
Fig. 11 Maps of estimated cortical activation differences between pleasant, unpleasant and neutral pictures in the time window from $350-450 \mathrm{~ms}$ after picture onset. Shown is the interpolated difference of grand means of estimated cortical activity $\left(\mathrm{nAm} / \mathrm{cm}^{2}\right)$ evoked by unpleasant minus pleasant or neutral pictures, respectively (modulus of cortical activity at each source dipole) projected onto a smoothed standard brain

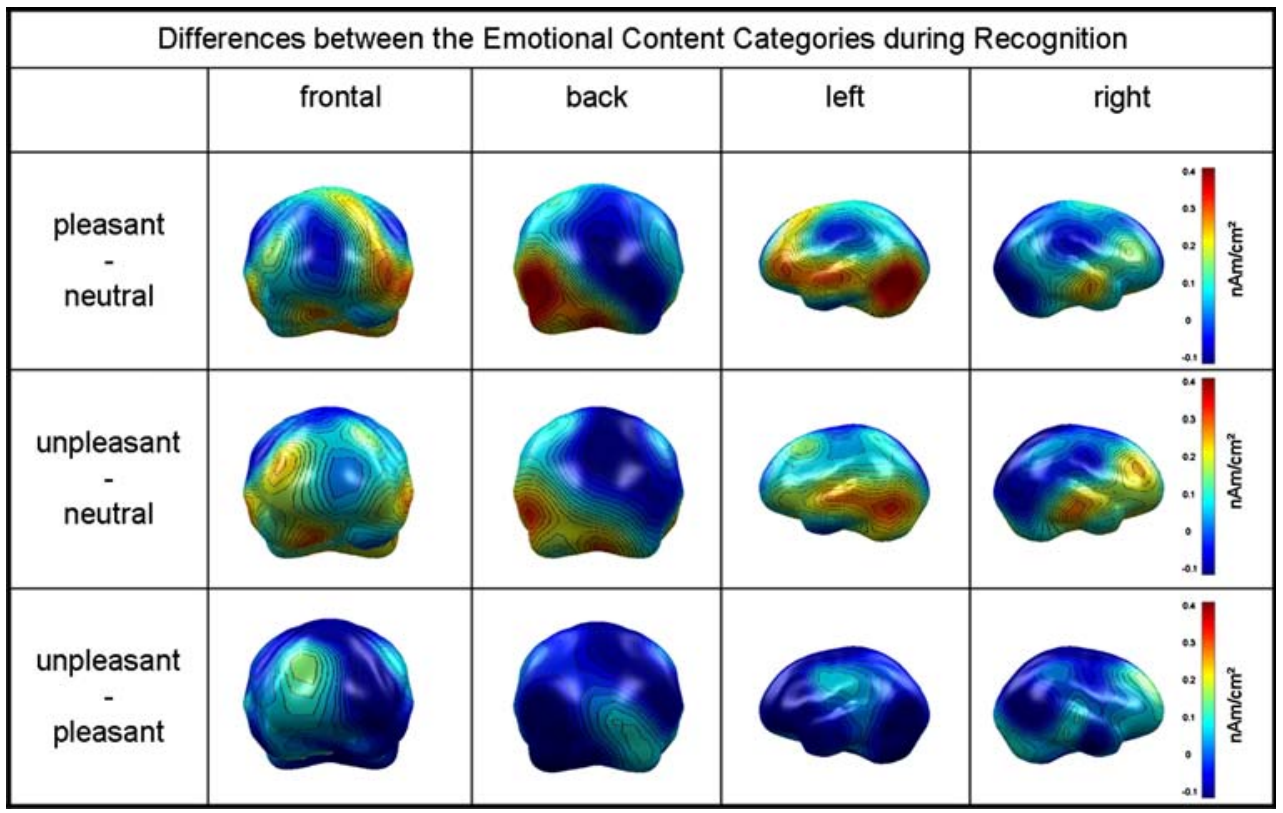


evoked neuromagnetic equivalents of the old-new effect. In line with previous ERP studies [19, 24, 34], a long lasting old-new effect was found. As previously reported (e.g. [24]), it was initially (350-450 ms) most pronounced in frontal brain regions, additionally, and unlike in previous EEG studies, it could also be observed in temporal regions. In the initial time window (about 350-450 ms) an interaction of emotional valence with stimulus repetition emerged, the old-new effect being most pronounced for pleasant pictures, thus paralleling the behavioural data. The early part of the old-new effect is thought to reflect familiarity-based judgements (see [34]) and therefore seems to reflect the neural counterpart of the behaviourally observed response bias in favour of pleasant pictures.

In the subsequent time-window (450-580 ms) the oldnew effect continued, now reaching significance in all analyzed brain regions, although inspection of the F-maps still suggests a fronto-temporal predominance. Emotional content ceased to modulate the old-new effect in this time window, but a main effect of emotional picture content emerged: Both pleasant and unpleasant pictures led to higher brain activities particularly in left temporal regions. In right temporal regions the effect was restricted to unpleasant pictures. This hemispheric asymmetry is at least partly in line with the assumption of a hemispheric asymmetry in emotional processing, with left brain structures preferentially involved in the processing of pleasant and right brain structures preferentially involved in the processing of unpleasant stimuli (e.g. [5]). Inspection of the F-maps additionally suggest enhancement frontal in response to both old and new emotional pictures, but this effect was more short lived and did not reach significance in the ANOVA analysis. The emotion effect is unrelated to picture recollection and may reflect unspecific attentional mobilization by emotional pictures, or, because of its relatively late on-set, enhanced evaluation and re-encoding processes of old and new emotional pictures alike. Although no further effects of emotional picture content were found, the old-new effect lasted for another $400 \mathrm{~ms}$, gradually decreasing in intensity. Its main cortical activation shifted to posterior brain regions (see Figs. 6 and 9).

Comparing the present MEG results with previous ERP data, irrespective of emotion modulation, there is a relative accentuation of frontal and temporal activity in comparison to parietal contributions to the old-new effect. This may on the one hand be due to a suggested greater accentuation of frontal processes (and possibly familiarity based decisions) when pictorial stimuli are used [17]. On the other hand, and in view of a large centro-parietal old-new effect recently found in a recognition memory study using complex scenes [19], this finding may reflect differential sensitivity of EEG and MEG to radial versus tangential generators. Particularly the later parietal parts of the old-new effect are thought to largely depend on deep medio-temporal structures $[14,52]$. If so, and if particularly radial generators project to the parietal lobe, such activity may be attenuated in the MEG. Conversely, tangential sources contributing to temporal lobe activity may be accentuated.

The present behavioural and neuromagnetic evidence concerning the effect of emotion on picture recognition run in parallel and are consistent with the view that recognition of pleasant material is substantially contributed to by familiarity judgements [24, 36]. It also comes as no surprise that apparent recognition advantages for emotionally arousing material are considerably attenuated or vanish when false alarms are taken into account [24, 53]. In fact, from an evolutionary point of view, which is often called upon when effects of emotion on information processing are discussed, a more liberal response criterion versus potentially relevant scenes and episodes may be more advantageous than maximally accurate discriminative recollection: It's often a certain type of stimulus rather than a particular instance that determines the response required. However, the, at least to some readers, more surprising aspect may be that in the present an emotional response bias persists for pleasant material. Our subjects were faster at deciding on pleasant pictures and they were also somewhat more willing to do so. Stimulus arousal cannot be the determining factor, since, if anything, unpleasant pictures were more arousing than pleasant ones, not the other way around. However, faster responses to pleasant stimuli are in line with the assumption that pleasant stimuli facilitate the approach system [28]. Faster reaction times when making recognition decisions on pleasant pictures have already been reported in Bradley et al. [2] and a number of recent studies have shown faster responses to pleasant stimuli in choice reaction tasks, particularly when affirmative button presses were required $[31,35,51]$. If indeed the response bias in favour of pleasant pictures at present is mediated by familiarity, there is clearly the possibility that 'real-life familiarity' determined this response bias. Although, as Ochsner [36] puts it, 'a chocolate brownie may not determine happiness in quite the same way as a snakebite could determine unhappiness', chocolate brownies might be more familiar to many, at least western people. Most of our experimental subjects will on average have had more reallife experience with the scenes depicted in the pleasant pictures (sports, family life, sex) than with the contents of the unpleasant pictures (crime and war scenes, mutilations, animal attack). Furthermore, appraisal of personal significance has been suggested to be a major factor influencing remembering [6, 36] and although impossible to assess post-hoc, it is also conceivable that our subjects implicitly found the pleasant material more self-relevant, positive affect being the modal rather than the exceptional experience in most people [9]. 
In sum, the present study delineated the time course and cortical distribution of neuromagnetic activity during recognition of pictures varying in emotional content. Large and protracted effects of picture recognition as well as effects of emotional content and an interaction of picture recognition with, albeit solely pleasant, emotional content, were identified and could be assigned to large-scale changes in regional cortical activity. On a behavioural level, parallel effects were found. The presently used data analysis approach gives an estimate of the spatio-temporal dynamics of the cortical events. Future studies may refine and complement this analysis with a realistic head model, taking into account individual anatomy and with source analysis procedures that offer 3D source estimation. More refined experimental designs may shed further light onto the functional significance of various aspects of recognition memory and its modulation by emotion.

\section{References}

1. Berg P, Scherg M. A multiple source approach to the correction of the eye artifacts. Electroencephalogr Clin Neurophysiol. 1994;90:229-41.

2. Bradley MM, Lang PJ. Measuring emotion: Self-assessment manikin and the semantic differential. J Behav Ther Exp Psychiatry. 1994;25:49-59.

3. Bradley MM, Greenwald MK, Petry MC, Lang PJ. Remembering pictures: pleasure and arousal in memory. J Exp Psychol Learn Mem Cogn. 1992;18:379-90.

4. Bradley MM, Moulder B, Lang PJ. When good things go bad: the reflex physiology of defense. Psychol Sci. 2005;16:468-73.

5. Canli T, Desmond JE, Zhao Z, Glover G, Gabrieli JD. Hemispheric asymmety for emotional stimuli detected with fMRI. Neuroreport. 1998;9: 3233-9.

6. Conway MA. Dewhurst SA. Remembering, familiarity, and source monitoring. Q J Exp Psychol A. 1995;48:125-40.

7. Cuthbert BN, Bradley MM, Lang PJ. Probing picture perception: activation and emotion. Psychophysiology. 1996;33:103-11.

8. Danion JM, Kauffmann-Muller F, Grangé D, Zimmermann MA, Greth P. Affective valence of words, explicit and implicit memory in clinical depression. J Affect Disord. 1995;34:227-34.

9. Diener E, Diener C. Most people are happy. Psychol Sci. 1996;7:181-5.

10. Dolan RJ. Emotion, cognition, and behavior. Science. 2002;298:1191-4.

11. Dolcos F, Cabeza R. Event-related potentials of emotional memory: encoding pleasant, unpleasant, and neutral pictures. Cogn Affect Behav Neurosci. 2002;2:252-63.

12. Dolcos F, LaBar KS, Cabeza R. Interaction between the amygdala and the medial temporal lobe memory system predicts better memory for emotional events. Neuron. 2004;42:855-63.

13. Dolcos F, LaBar KS, Cabeza R. Remembering one year later: role of the amygdala and the medial temporal lobe memory system in retrieving emotional memories. Proc Natl Acad Sci U S A. 2005;102:2626-31

14. Düzel E, Vargha-Khadem F, Heinze HJ, Mishkin M. Brain activity evidence for recognition without recollection after early hippocampal damage. Proc Natl Acad Sci U S A. 2001;98:8101-6.

15. Düzel E, Habib R, Schott B, Schoenfeld A, Lobaugh N, McIntosh AR, et al. A multivariate, spatiotemporal analysis of electromagnetic time-frequency data of recognition memory. Neuroimage. 2003;18:185-97.

16. Düzel E, Habib R, Guderian S, Heinze HJ. Four types of noveltyfamiliarity responses in associative recognition memory of humans. Eur J Neurosci. 2004;19:1408-16.

17. Friedman D. Cognitive event-related potential components during continuous recognition memory for pictures. Psychophysiology. 1990;27:136-48.

18. Graham R, Cabeza R. Event-related potentials of recognizing happy and neutral faces. Neuroreport. 2001;12:245-8.

19. Gutchess AH, Ieuji Y, Federmeier KD. Event-related potentials reveal age differences in the encoding and recognition of scenes. J Cogn Neurosci. 2007;19:1089-103.

20. Hamalainen MS, Ilmoniemi RJ. Interpreting magnetic fields of the brain: minimum norm estimates. Med Biol Eng Comput. 1994;32:35-42.

21. Hauk O. Keep it simple: a case for using classical minimum norm estimation in the analysis of EEG and MEG data. Neuroimage. 2004;21:1612-21.

22. Hauk O, Keil A, Elbert T, Müller MM. Comparisons of the data transformation procedures to enhance topographical accuracy in time-series analysis of the human EEG. J Neurosci Methods. 2002;113:111-22.

23. Inaba $M$, Nomura $M$, Ohira $H$. Neural evidence of effects of emotional valence on word recognition. Int $\mathbf{J}$ Psychophysiol. 2005;57:165-73.

24. Johansson M, Mecklinger A, Treese AC. Recognition memory for emotional and neutral faces: an event-related potential study. J Cogn Neurosci. 2004;16:1840-53.

25. Junghofer M, Peyk P. Analyse und Visualisierung von Hirnstrom- und Hirnmagnetfeld-Messungen. [Analysis of electrical potentials and magnetic fields of the brain]. Matlab Select 2004;2:24-8. EMEGS software is freely available at http:// www.emegs.org/.

26. Junghofer M, Bradley MM, Elbert TR, Lang PJ. Fleeting images: a new look at early emotion discrimination. Psychophysiology. 2001;38:175-8.

27. Kissler J, Herbert C, Peyk P, Junghofer M. Buzzwords: early cortical responses to emotional words during reading. Psychol Sci. 2007; 18:475-80.

28. Lang PJ, Bradley MM, Cuthbert BN. Emotion, attention, and the startle reflex. Psychol Rev. 1990;97:377-95.

29. Lang PJ, Bradley MM, Cuthbert BN. International affective picture system (IAPS): Affective ratings of pictures and instruction manual. Technical Report A-6. Gainesville, FL. 2005.

30. Leiphart J, Rosenfeld JP, Gabrieli JD. Event-related potential correlates of implicit priming and explicit memory tasks. Int $\mathbf{J}$ Psychophysiol. 1993;15:197-206.

31. Leppänen JM, Hietanen JK. Positive facial expressions are recognized faster than negative facial expressions, but why? Psychol Res. 2004;69:22-9.

32. Maratos EJ, Rugg MD. Electrophysiological correlates of the retrieval of emotional and non-emotional context. J Cogn Neurosci. 2001;13:877-91.

33. Maratos EJ, Allan K, Rugg MD. Recognition memory for emotionally negative and neutral words: an ERP study. Neuropsychologia. 2000;38:1452-65.

34. Mecklinger A. Interfacing mind and brain: a neurocognitive model of recognition memory. Psychophysiology. 2000;37: 565-82.

35. Neumann R, Strack F. Approach and avoidance: the influence of proprioceptive and exteroceptive cues on encoding of affective information. J Pers Soc Psychol. 2000;79:39-48.

36. Ochsner KN. Are affective events richly recollected or simply familiar? The experience and process of recognizing feelings past. J Exp Psychol Gen. 2000;129:242-61. 
37. Oldfield RC. The assessment and analysis of handedness: The Edinburgh inventory. Neuropsychologia. 1971;9:97-113.

38. Palomba D, Angrilli A, Mini A. Visual evoked potentials, heart rate responses and memory to emotional pictorial stimuli. Int $\mathrm{J}$ Psychophysiol. 1997;27:55-67.

39. Perlstein WM, Elbert T, Stenger VA. Dissociation in human prefrontal cortex of affective influences on working memoryrelated activity. Proc Natl Acad Sci U S A. 2002;99:1736-41.

40. Richardson MP, Strange BA, Dolan RJ. Encoding of emotional memories depends on amygdala and hippocampus and their interactions. Nat Neurosci. 2004;7:278-85.

41. Rugg MD, Allan K. Memory retrieval: An electrophysiological perspective. In: Gazzaniga MS, (editors). The new cognitive neurosciences. 2nd ed. Cambridge: MIT Press; 2000.p. 805-16.

42. Schupp HT, Cuthbert BN, Bradley MM, Cacioppo JT, Ito T, Lang PJ. Affective picture processing: the late positive potential is modulated by motivational relevance. Psychophysiology. 2000;37:257-61.

43. Schupp HT, Junghofer M, Weike AI, Hamm AO. Emotional facilitation of sensory processing in the visual cortex. Psychol Sci. 2003;14:7-13.

44. Schupp HT, Stockburger J, Codispoti M, Junghofer M, Weike AI, Hamm AO. Selective visual attention to emotion. J Neurosci. 2007;27:1082-9.

45. Smith AP, Dolan RJ, Rugg MD. Event-related potential correlates of the retrieval of emotional and nonemotional context. $\mathrm{J}$ Cogn Neurosci. 2004;16:760-75.
46. Snodgrass JG, Corwin J. Pragmatics of measuring recognition memory: applications to dementia and amnesia. J Exp Psychol Gen. 1988;117:34-50.

47. Staresina BP, Bauer H, Deecke L, Walla P. Magnetoencepahlographic correlates of different levels in subjective recognition memory. NeuroImage. 2005;27:83-94.

48. Tendolkar I, Rugg M, Fell J, Vogt H, Scholz M, Hinrichs H, et al. A magnetoencephalographic study of brain activity related to recognition memory in healthy young human subjects. Neurosci Lett. 2000;280:69-72.

49. Van Petten C, Senkfor AJ. Memory for words and novel visual patterns: repetition, recognition, and encoding effects in the event-related brain potential. Psychophysiology. 1996;33:491506.

50. Walla P, Hufnagl B, Lindinger G, Deecke G, Imhof L, Lang W. False recognition depends on depth of prior word processing: a (MEG) study. Brain Res Cogn Brain Res. 2001;11:249-57.

51. Wentura D. Dissociative affective and associative priming effects in lexical decision task: yes versus no responses to word targets reveal evaluative judgment tendencies. J Exp Psychol Learn Mem Cogn. 2000;26:456-69.

52. Wilding EL, Rugg MD. An event-related potential study of recognition memory with and without retrieval of source. Brain. 1996;119(Pt 3):889-905.

53. Windmann S, Kutas M. Electrophysiological correlates of emotion-induced recognition bias. J Cogn Neurosci. 2001;13:577-92. 